\title{
La herramienta de transparencia en las noticias sobre la COVID-19 publicadas por el Diario Público durante los primeros 14 días del estado de alarma
}

\author{
NÀdia Alonso-López \\ Universitat Politècnica de València \\ naallo1@har.upv.es \\ https://orcid.org/0000-0002-5220-2232
}

\author{
Raúl TeRol-Bolinches \\ Universitat Politècnica de València \\ rautebo@upv.es \\ https://orcid.org/0000-0002-0915-7288
}

\section{The transparency tool in the news about COVID-19 published by Diario Público during the first 14 days of the State of Alarm}

\section{RESUMEN ABSTRACT}

En la Era de la Posverdad cada vez es más complicado discernir entre aquellas informaciones que son reales y las que

no. La proliferación de las noticias falsas ha llevado a los medios convencionales a mostrar el proceso de verificación de las informaciones publicadas, así como a la aparición de agencias de verificación de la información, en aras de una mayor transparencia y credibilidad. Con el inicio de la

pandemia provocada por la COVID-19 el volumen de los bulos difundidos por las redes sociales y las aplicaciones de mensajería instantánea se incrementó de manera exponencial, con lo que se hizo más necesario el compromiso de veracidad de las informaciones publicadas en los medios. La presente investigación tiene como objetivo principal analizar el índice de transparencia de las noticias relacionadas con la COVID-19 y que fueron publicadas en el primer tramo del estado de alarma, los primeros 14 días tras el anuncio del presidente Pedro Sánchez hasta su primera renovación en el congreso. Tras un estudio previo, se ha optado analizar las informaciones del diario Público, que es el único que ofrece una herramienta de transparencia que muestra la trazabilidad de sus informaciones, relacionándolas con algunos ítems o palabras clave.

\section{PALABRAS CLAVE}

Noticias falsas; COVID-19; Posverdad; Transparencia; Diario Público.
In the Post-Truth Era, it is increasingly difficult to discern between information that is real and information that is not. The proliferation of fake news has led the conventional media to show the process of data verification of published information, as well as the emergence of information verification agencies. With the onset of the pandemic caused by COVID-19 the volume of hoaxes spread by social networks and instant messaging applications increased exponentially. The present research has as its main objective the analysis of the transparency index of the news related to COVID-19 and that were published in the first stretch of the state of alarm, the first 14 days after the announcement of President Pedro Sánchez until its first renewal in Congress. After a previous study, it has been chosen to analyze the information of the newspaper Público, which is the only one that offers a transparency tool that shows the traceability of its information and has been related to some items or keywords.

KEYWORDS

Fake news; COVID-19; Post-truth; Transparency; Diario Público. 


\section{Introducción}

La facilidad con la que se accede a la información en nuestros días se debe, entre otros factores, a la elevada penetración del smartphone en nuestra sociedad, que se ha producido de una manera exponencial a lo largo de los últimos años (Pérez-Escoda, 2018). A ello se añaden aspectos como el carácter gratuito, de publicación y actualización inmediata, la ubicuidad o el permanente acceso a las informaciones en el actual ecosistema mediático que favorecen el crecimiento del consumo de las noticias a través de las ediciones digitales de los periódicos o de los llamados nativos digitales (García Avilés, 2017). La tecnología ha facilitado que el proceso de comunicación se produzca de manera más sencilla, instantánea y accesible (Culver y Jacobson, 2012). Por otra parte, la crisis sanitaria global provocada por la COVID-19 ha generado muchos cambios en nuestro comportamiento social. Con el fin de evitar contagios, se han modificado muchos de los hábitos de la sociedad. Por ejemplo, el hecho de tener que permanecer en los hogares como consecuencia del confinamiento domiciliario durante el primer estado de alarma decretado en España en marzo de 2020 indujo a un aumento en el acceso a la información digital sobre cuestiones relacionadas con la pandemia (Abuín-Vences et ál., 2020; Casero-Ripolles, 2020).

Según un estudio sobre el uso de las redes sociales realizado por IAB Spain (2020), un 93\% de la población tiene acceso a Internet y un 66\% utiliza alguna de las diferentes redes sociales con el fin de estar informado. Los medios de comunicación se sitúan en cuarta posición con un $41 \%$ en cuanto a seguimiento por parte de la ciudadanía, solo por detrás del círculo de familiares y allegados, influencers o marcas. En este contexto, España es el país en el que más preocupan los bulos y la desinformación; a más de un 60\% le inquieta no saber diferenciar entre una información veraz y otra falaz, así como también es el país en el que los usuarios de la información online no se limitan a su consumo a través de la red, sino que, además, interactúan con ella, tal y como se refleja en el Digital News Report 2020 (Negredo et al., 2020). Estos datos refuerzan el incremento en el consumo de información durante el inicio de la crisis sanitaria global en la que, además, empezó a circular una gran cantidad de información sobre la COVID-19 y sus efectos por diferentes plataformas, generando paralelamente en el ámbito comunicativo lo que se conoció con el nombre de "Infodemia" (OMS, 2020).

Mezclada con esa información y con un aspecto similar, aunque con fines completamente distintos, dentro de ese océano de datos que es Internet se pueden encontrar bulos o noticias falsas con el claro objetivo de desinformar a la población y sembrar la duda o incluso el pánico en muchas ocasiones en un ecosistema en el que cualquiera puede convertirse en productor de la información y dirigirse a una audiencia global (Bergström y Jervelycke, 2018). Se trata de noticias que gozan de una mayor aceptación que una información veraz y que tienen una gran capacidad para convertirse en virales entre los usuarios, llegando hasta a un $70 \%$ de posibilidades de ser compartidas por una persona real y no por un bot (Silverman et al., 2016; Dizikes, 2018; Orbegozo-Terradillos et al., 2020).

El escenario de la comunicación actual favorece la información equívoca, sin ninguna precisión y con malas intenciones, en muchos casos producto de la inventiva, y cuyo objetivo es inducir al error a los usuarios e intoxicarles en el proceso de información (Zommer, 2014; Ufarte, Anzera y Murcia, 2020).

\subsection{Una aproximación al concepto de Fake News}

La Era de la Posverdad (Keyes, 2004) ha situado al periodismo en uno de los momentos más delicados de su historia, con una confianza en decadencia consecuencia del elevado número de informaciones falsas o fake news que circulan por Internet y que, en ocasiones, cuesta discernir entre si son o no noticias reales. El término Posverdad nos conduce a reflexionar sobre qué es verdad y qué es mentira. Esta consideración desde el ámbito periodístico pasa por la verificación de las fuentes y el contraste de datos a la hora de publicar una noticia, no dejando lugar a que exista la mínima posibilidad a la vacilación (Nigro, 2018), aunque a pesar de ello, en el panorama actual nos encontramos con que se utiliza la desinformación deliberadamente (Ufarte, Sidorenko y Cantero, 2019). En esta Era nos encontramos con que la desinformación y las fake news significan una amenaza de gran relevancia para la sociedad actual, generando gran incertidumbre entre la ciudadanía y temiendo que su efecto negativo pueda hacer zozobrar los pilares de la democracia (Stewart et ál., 2019; European Commission, 2018a).

Podríamos definir posverdad como un "marco de referencia más amplio que las fake news (...), una idea, un imaginario, un conjunto de representaciones sociales o sentidos ya incorporados por las audiencias y desde donde son posibles fake news que refieren a esa idea afirmándola o ampliándola" (Murolo, 2019, p. 68). Están estrechamente relacionados con la Posverdad el declive de los medios convencionales y el crecimiento de las plataformas de los conocidos como "falsos medios hiperpartisanos" (Aparici y García-Marín, 2019).

En cuanto a la definición del término fake news y a las diferentes tipologías de noticias falsas, se debe considerar este concepto como aquellas informaciones llamativas que han sido concebidas para su difusión a través de las redes sociales (Pennycock y Rand, 2018) y cuyo contenido tiene grandes similitudes con el que podríamos ver en los medios de comunicación por lo que a su forma respecta, pero que difiere en el proceso o en la intencionalidad que tiene (Lazer et al., 2018). Amorós-García (2018) se refiere a las fake news como las informaciones falsas que se disfrazan con el aspecto de noticias reales y cuya finalidad no es otra que la de confundir a los lectores y propagar una falsedad o desinformación mediante 
la que se obtiene beneficio político o de otra índole. A todo ello se podrían añadir otras utilizaciones para este concepto, como la cobertura de noticias con aire tendencioso, el uso de retóricas arbitrarias o el empleo de falsos testimonios protagonistas (Nielsen y Graves, 2017). La Comisión Europea las define como "información falsa, inexacta o engañosa diseñada, presentada y promovida para causar daño público intencionalmente o con fines de lucro" (European Commission, 2018a).

Por lo que respecta a las diferentes tipologías de fake news, hay dos elementos que son comunes a las diferentes aportaciones académicas que se han realizado sobre este objeto de estudio: la intencionalidad que las motiva y la desinformación que originan (Orbegozo-Terradillos et al., 2020). Tandoc, Lim y Ling (2018) señalan una taxonomía de las fake news basada en dos variables: el grado de autenticidad y el de engaño. A partir de ahí establecen cinco tipos de noticias falsas: la noticia satírica, la parodia, las noticias fabricadas, la manipulación fotográfica, la publicidad y las relaciones públicas y, por último, la propaganda.

En un estudio reciente se recomienda la utilización del vocablo "bulo" para referirse a las fake news, evitando de esta manera el uso innecesario de anglicismos ofreciendo una precisión significativa. En ese mismo trabajo, los autores definen bulo como "todo contenido intencionadamente falso y de apariencia verdadera, concebido con el fin de engañar a la ciudadanía, y difundido públicamente por cualquier plataforma o medio de comunicación social" (Salaverría et al., 2020, p. 12). Además de definir el término, realizan una descripción sobre las tipologías de los bulos: bromas, exageraciones, descontextualizaciones y engaños. Por otro lado, en un informe publicado en 2017, se recomendaba la no utilización del término fake news por considerar que describe de manera inadecuada el enmarañado proceso de la información contaminada, así como también por el mal uso que políticos y gobernantes de distintas partes del mundo han hecho para atacar aquellos periodistas o medios de comunicación que no son de su agrado, decantándose por la palabra desinformación (Derakhshan y Wardle, 2017).

\section{La importancia de la transparencia en la información}

Transparencia se define como "la apertura de información por parte de las instituciones públicas y de otras entidades sin restricciones, de forma que los datos que se aporten sean fiables y actualizados para que la ciudadanía pueda tomar decisiones responsables" (Díez-Garrido, Campos-Domínguez, Calvo, 2019, p. 85). La transparencia en España no ha sido un elemento fundamental en las instituciones o en las empresas de comunicación, prueba de ello es que hasta 2013 no queda regulada mediante una ley cuyos objetivos principales son los de asegurar que toda la ciudadanía pueda ejercer su derecho de acceso a la información, exigir la transparencia en las administraciones públicas y controlar que se realicen prácti- cas de gobernanza justas (Applegren y Salaverría, 2018).

Por lo que respecta a las empresas informativas, se puede comprender la transparencia como el hecho de difundir públicamente aquella información relevante relativa a los medios de comunicación en los ámbitos de la estructura, la organización y la propiedad, la situación financiera y aquellos vínculos económicos y políticos que puedan existir (Heikkilä et ál., 2012). Hace referencia, a su vez, al compromiso que tienen los medios de comunicación de explicar tanto el proceso editorial como las prácticas profesionales que se han seguido (Diakopoulos y Koliska, 2017). Así como también conlleva desvelar cuáles han sido las interacciones que el medio ha mantenido con su público (Campos-Domínguez y Redondo-García, 2015). Se puede fomentar la transparencia mediante herramientas de rendición de cuentas que posibiliten al consumidor de la información poder evaluar la calidad del proceso informativo o del producto mediático que se consume (Fangler et ál., 2014). Las empresas de comunicación deben implementar elementos diversos que sirvan para la monitorización de la participación y proteger la calidad de las informaciones, ya sea en sus páginas web como en sus perfiles en las principales redes sociales (Suárez-Villegas, Rodríguez-Martínez y Ramón-Vegas, 2020)

Este trabajo se centra en el análisis del indicador de transparencia que ofrece la herramienta TJ Tool del Diario Público, que es su creador y el único periódico generalista hasta el momento en implementar este tipo de innovación. Su nombre proviene del acrónimo Transparent Journalism, y su principal objetivo es generar confianza entre sus lectores y acabar con las fake news de una manera eficaz (Mapa de transparencia, 2020). Esta aplicación busca un aumento en la transparencia informativa mediante la trazabilidad de aquellas informaciones de carácter periodístico que componen la noticia, ofreciendo transparencia a lo largo del proceso informativo. Con un total de ocho indicadores de análisis, la herramienta TJ Tool nos ofrece un valor numérico que tiene que ver con el grado en que cada uno de ellos se cumple a la hora de elaborar la noticia (Terol-Bolinches y Alonso-López, 2020). La herramienta está abierta a su uso por parte de cualquier periodista o medio de comunicación que quiera integrarla en su página web para ofrecer este servicio a sus usuarios y favorecer la generación de confianza en el medio periodístico (Sánchez, 2019).

El diario Público, siguiendo las recomendaciones de una serie de organismo internacionales vinculados a la comunicación y la información, decide poner en funcionamiento esta herramienta con la finalidad de poder lograr una mejor trazabilidad de la información y que se hagan públicos los procesos informativos, para que sus usuarios confíen en el rigor del medio que han elegido para informarse. TJ Tool realiza un rastreo prácticamente automático de los ocho indicadores que se recogen: fuentes; documentos de apoyo; contexto; fecha; lugar de redacción; autor; motivo editorial y política de transparencia (Mapa de transparencia, 2020). Para el acceso a este conte- 
nido el usuario debe pulsar la letra $T$, que se encuentra junto con los iconos de las redes sociales o en la esquina superior derecha del sitio web. A pesar de haber sido desarrollada por el propio diario, queda explícito que cualquier otra empresa informativa podría utilizar esta aplicación para la verificación de las informaciones publicadas por su medio (Terol-Bolinches y Alonso-López, 2020).

Hasta el momento, se había asistido a la verificación de datos por medio de agentes independientes -Newtral o Maldita- o departamentos de agencias de noticias como Efe Verifica. En este sentido, los verificadores de datos pueden contribuir a que se reduzca el grado de desinformación, aunque entre sus posibilidades no se encuentra la de reparar el daño que se causa mediante estos bulos o informaciones falsas (Nyhan y Reifler, 2012). De esta manera, el auge del fact-checking o la incorporación de las herramientas de trazabilidad de la información implementadas por las empresas de comunicación y los diarios digitales favorecerán la mejora en la confianza y credibilidad de los usuarios en el medio (Ireton y Posetti, 2018; García-Galera, et al., 2020; Terol-Bolinches y Alonso-López, 2020). Por lo que se refiere a la difusión de informaciones falsas relacionadas con el ámbito científico y la salud, son propagadas con una mayor facilidad a través de las redes sociales como consecuencia del grado de dificultad a la hora de ser contrastadas (Molina-Cañabate y Magallón-Rosa, 2020).

\subsection{La verificación de datos en el periodismo}

Se puede definir la verificación de datos, o fact-checking, como la herramienta que mejor se adecúa en la lucha contra las informaciones falsas (Amorós, 2018) creada desde el periodismo con la finalidad de ofrecer un mayor rigor en las informaciones vertidas. Mantzarlis (2018) apunta que se trata de la comprobación de toda la serie de informaciones y datos que circulan por la red, con verificaciones de todo aquello que ha sido dicho por los protagonistas y aquello que pueda ser noticioso.

Fue en los primeros años del siglo pasado, concretamente en 1913, cuando se crea por primera vez una práctica desde una organización que es el origen de la verificación de datos. Se trata de la fundación del Bureau Accuracy and Fair Play por el diario New York World. Este organismo se dedicaba principalmente a corregir aquellas informaciones que no eran precisas del todo y a suprimir las que eran falsas (Ayuso y Bauzá, 2018). También en esas primeras décadas, revistas estadounidenses como Time ya aplicaban un proceso de comprobación de la información antes de publicar las noticias (Fabry, 2017). El objetivo era exigir responsabilidades a aquellos que ejercían la función pública y ofrecer una información de calidad a la ciudadanía, aspectos fundamentales para el fortalecimiento de la democracia (Gueham, 2017). De hecho, si se pone el foco en nuestros días, varios han sido los países en los que el debate político se ha centrado en torno al tema de la desinformación durante la pandemia provocada por la COVID-19 (Salaverría et ál., 2020).

En este sentido, la práctica de la verificación de datos se ha extendido y se han puesto en marcha diversas iniciativas desde las empresas informativas dedicadas a esta función (Palau-Sampio, 2015). El número de proyectos de verificación de datos activos en todo el mundo supera los 300, un centenar más de lo que se había registrado el año anterior en ese mismo estudio (Duke Reporter's Lab, 2020). Como se puede apreciar en el informe, son muchos los medios de comunicación y las plataformas de carácter independiente que han decidido poner en marcha secciones, grupos de trabajo o espacios enfocados en la verificación de las informaciones publicadas por otros medios y distribuidas a través de las redes sociales (Cherubini y Graves, 2016). La primera de las medidas que se proponen por el grupo de expertos consultado por la Comisión Europea para combatir la desinformación pasa por la mejora de la transparencia de las noticias online (European Commission, 2018b), aspecto por el que destaca el objeto de estudio de esta investigación. Las cuatro medidas restantes son la promoción de una alfabetización mediática o digital (Molina-Cañabate y Magallón-Rosa, 2020); el desarrollo de herramientas con las que capacitar a los usuarios y periodistas para afrontar la desinformación; la protección de la diversidad y sostenibilidad del ecosistema mediático; y la promoción de la investigación continua sobre el impacto de la desinformación en Europa (European Commission, 2018b).

En la actualidad, gracias a las nuevas tecnologías y a la eclosión de las fake news, se han llevado a cabo una serie de sistemas de verificación de la información desde tres ámbitos bien diferenciados: el periodismo, la academia y distintos entes gubernamentales (Magallón, 2018). La evolución de estos verificadores en los últimos años ha sido muy acelerada, hecho que ha dado lugar a que se consolide la International Fact-Checking Network (IFCN), ubicada en el Poynter Institute for Media Studies. Es a partir de 2008, con las elecciones presidenciales de los Estados Unidos, cuando se empieza a desarrollar el formato de verificación digital en Internet, aunque encontramos algún referente anterior como pueda ser el caso de Factcheck.org, que en 2003 vio la luz en el seno del Annenberg Public Policy Center de la University of Pennsylvania (EE.UU.), o la herramienta que puso en marcha el periódico The Washington Post en el año 2007 (Molina-Cañabate y Magallón-Rosa, 2020) o Le Monde en 2009 (Ramon-Vegas et al., 2020).

\section{Objetivos y metodología}

El presente estudio plantea como objetivo principal estudiar si se establece una relación entre el indicador de transparencia de las noticias relacionadas con la COVID-19 publicadas en la sección de Política del Diario Público.es durante el primer período del estado de alarma, comprendido entre el 14 y el 27 
LA CRISIS DEL CORONAVIRUS

\section{España cierra sus fronteras terrestres salvo a españoles, residentes y trabajadores transfronterizos}

"A partir de las 12 horas de esta noche, solo podrán entrar ciudadanos españoles", dijo Marlaska. "Las restricciones de entrada no tendrán efecto en el transporte de mercancías", añadió el ministro, en rueda de prensa desde La Moncloa.

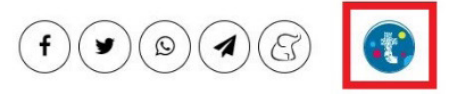

Figura 1. Cabecera de noticia con icono de la herramienta de transparencia. Fuente: Publico.es. Elaboración propia.

de marzo de 2020 y determinados parámetros que se detallan a continuación.

Como objetivos específicos se establecen:

- Determinar la relación entre el indicador de transparencia y los parámetros analizados.

- Identificar el resultado del indicador de transparencia obtenido en más o menos ocasiones en las noticias analizadas.

- Definir el porcentaje de informaciones que no disponen del indicador de transparencia y ponerlo en relación con los parámetros analizados.

En este sentido, se plantean las siguientes preguntas de investigación:

RQ1: ¿Qué porcentajes del indicador de transparencia se repiten con mayor y menor frecuencia en las noticias relacionadas con la COVID-19 en el periodo estudiado?

RQQ2: ¿Hay relación entre el indicador de transparencia de las noticias y los parámetros analizados?

RQ3: ¿Hay relación entre las noticias que no disponen del indicador de transparencia y los parámetros analizados?

La metodología empleada para la realización de este trabajo combina el método de investigación cuantitativo con el cualitativo de análisis de parámetros en relación con el indicador de transparencia proporcionado por la herramienta TjTool que implementa el diario Público para mostrar la trazabilidad de las informaciones que publica. Se opta por este diario porque, tal y como se concluye en una investigación previa (Terol-Bolinches y Alonso-López, 2020), es el único en España que aplica esta herramienta, la cual proporciona un indicador que muestra el porcentaje de transparencia de las noticias en una escala de 0 a $100 \%$.

A continuación, se delimita el objeto de estudio a las noticias de la sección de Política identificadas en la cabecera de las informaciones por el propio diario Público.es como "La crisis del Coronavirus", "Coronavirus", "Crisis del COVID-19". Asimismo, se concreta el período de tiempo a las dos semanas correspondientes al primer estado de alarma decretado por el Gobierno de España, entre el 14 y el 27 de marzo de 2020, como se ha indicado anteriormente. Se opta por este espacio de tiempo por tratarse de las primeras semanas de confinamiento de la población, un período de incertidumbre en el que aumenta de forma considerable el acceso a los medios de comunicación (Aleixandre-Benavent, Castelló-Cogollos y Valderrama-Zurián, 2020) y en el que, por tanto, resulta de gran relevancia discernir entre informaciones veraces y los "bulos" o "noticias falsas". En este caso, la aplicación de la herramienta TjTool promueve una definición de la transparencia de las informaciones publicadas para obtener así una mayor credibilidad para los usuarios del diario.

Atendiendo a estos criterios, se ha obtenido una muestra de 117 noticias de las que se adquiere el indicador de transparencia accediendo a través del propio diario Público.es en el icono situado a la derecha del titular en la parte inferior, que se puede observar en la figura 1.

Al clicar en este icono, se accede a la página con el resultado de dicho indicador, denominado mapa de transparencia, de lo que se observa un ejemplo en la figura 2. Conviene aclarar que no todas las noticias disponen del icono de esta herramienta o dan acceso a la misma, de cuyo porcentaje también se ocupa la presente investigación, como se especifica al final de este apartado.

Para el análisis del contenido de las noticias y su posterior relación con el indicador de transparencia, se han planteado 


\section{España cierra sus fronteras terrestres salvo a españoles, residentes y trabajadores transfronterizos}

Esta noticia tiene un valor de transparencia de 76 sobre un máximo de 100.

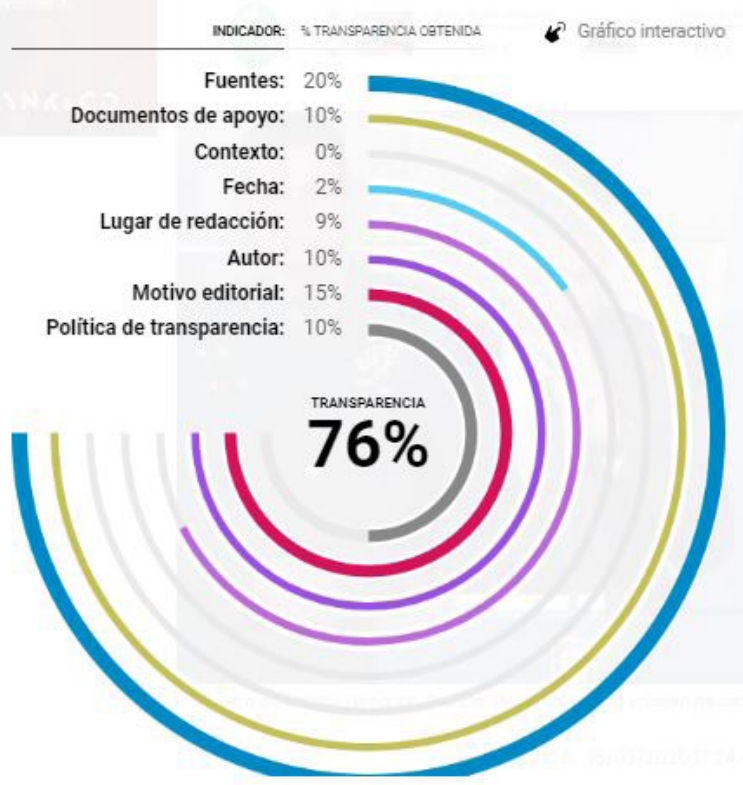

Figura 2. Mapa de transparencia de una noticia. Fuente: Publico.es

\begin{tabular}{|c|c|c|c|c|c|c|c|}
\hline \multirow[b]{2}{*}{ 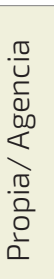 } & \multirow[b]{2}{*}{ 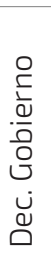 } & \multirow[b]{2}{*}{ 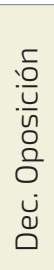 } & \multicolumn{5}{|c|}{ Veces que se repiten las palabras } \\
\hline & & & $\begin{array}{l}\text { Un } \\
\cdot \frac{1}{2} \\
\frac{1}{0} \\
0 \\
0\end{array}$ & $\stackrel{\ominus}{\ominus}$ & 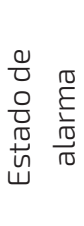 & 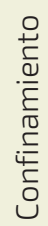 & 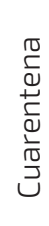 \\
\hline
\end{tabular}

Tabla 1. Parámetros de estudio. Elaboración propia.

Como se puede observar en la tabla, se localiza si las noticias son propias del diario Público o de Agencias y si contienen declaraciones de representantes del Gobierno de España y/o de los partidos de la Oposición, considerándose como tales las formaciones políticas que no apoyaron la investidura del gobierno tras las últimas elecciones. A continuación, se busca en cada una de las noticias analizadas las veces que aparecen las palabras Coronavirus, COVID, estado de alarma, confinamiento y cuarentena. Se han tomado estas palabras como referencia por tratarse de noticias relacionadas con la COVID-
Este es el resultado generado por la Calculadora de Transparencia de nuestra herramienta. Aqui puedes encontrar más información sobre los criterios de transparencia aplicados por la Herramienta de Periodismo Transparente de Público.

Esta noticia ha sido actualizada desde su creación. Para ver el Mapa de Transparencia de las actualizaciones anteriores, selecciona la versión que quieres consultar aqui:

Actualización de la noticia $\quad$ 16/03/2020 17:58 (Versión: 3001234) .

(

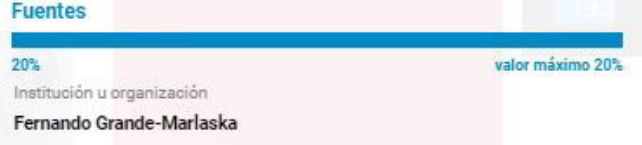

Tratado de Schengen

Persona

UE

(2)

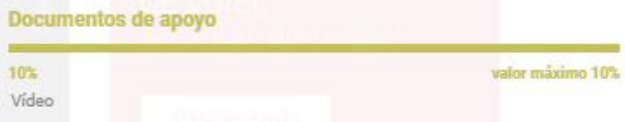

Renuncia nula: la argucia de Felipe VI con su herencia

Sánchez decreta el estado de alarma y la autoridad será el Gobierno

Imager

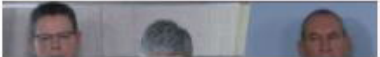

19 durante el estado de alarma y encontrarse entre las más buscadas en el período de análisis según Google Trends. Además, confinamiento fue declarada por la RAE como palabra del año 2020.

Una vez realizado el análisis, se relacionan los resultados con el índice de transparencia obtenido en cada noticia. Se trabaja con tablas de Excel para calcular los porcentajes de los parámetros y establecer las diferencias halladas entre los mismos y la relación con los indicadores de transparencia. Asimismo, se agrupan los indicadores de transparencia en intervalos, lo que permite observar los valores específicos que concentran desde una perspectiva más amplia para el análisis.

Además, como se ha indicado en líneas anteriores, se incluyen en el análisis las noticias que no contienen la herramienta TjTool así como las informaciones que no la tienen disponible, diferenciando entre ambas, con el fin de establecer también si se asocian los parámetros estudiados al hecho de no disponer del indicador de transparencia. 


\section{Resultados}

\subsection{Porcentajes del indicador de transparencia}

Atendiendo a la pregunta de investigación 1, en la muestra de 117 noticias analizadas no se encuentran informaciones cuyo indicador de transparencia sea del 0\% (mínimo) ni tampoco del 100\% (máximo). Concretamente, ninguna información analizada está por encima del $94 \%$ ni por debajo del $12 \%$ en cuanto al indicador de transparencia. En este sentido, cabe destacar que el $2 \%$ de las noticias analizadas alcanzan el porcentaje del $94 \%$ en el Indicador de transparencia, mientras que al 10\% de las noticias les corresponde el $12 \%$. De hecho, este indicador es el que aglutina el mayor porcentaje de las noticias analizadas junto al 76\%, indicador de transparencia resultante también en el 10\% de las informaciones objeto de estudio.

En cuanto a los indicadores de transparencia agrupados por intervalos, como se puede observar en el gráfico 1, la mayor parte de las noticias analizadas, en concreto un 34\%, disponen de un indicador de transparencia que se sitúa entre el 76 y el $100 \%$, aunque, como se ha mencionado, ninguna de ellas resulta con el $100 \%$ de transparencia. A continuación, el 30\% de las noticias obtienen un indicador de transparencia entre el 51 y el 75\%. Por otro lado, el indicador de transparencia resultante entre el 26 y el $50 \%$ es el que cuenta con un menor porcentaje de noticias, en concreto, el $2 \%$.

En el gráfico 1 también se puede observar que no todas las noticias disponen de indicador de transparencia. En el 13\% de las noticias analizadas no se encuentra la herramienta, mientras que en el 10\% de las mismas, la herramienta no está disponible. En la figura 3 se puede observar una muestra de una noticia que no cuenta con la herramienta de transparencia y en la figura 4, una noticia en la que, al clicar en el icono, responde que la herramienta no se encuentra disponible.

Por tanto, el 23\% de las noticias analizadas no proporcionan información sobre su indicador de transparencia, ya sea porque no se encuentra el icono, ya sea porque la herramienta no está disponible.

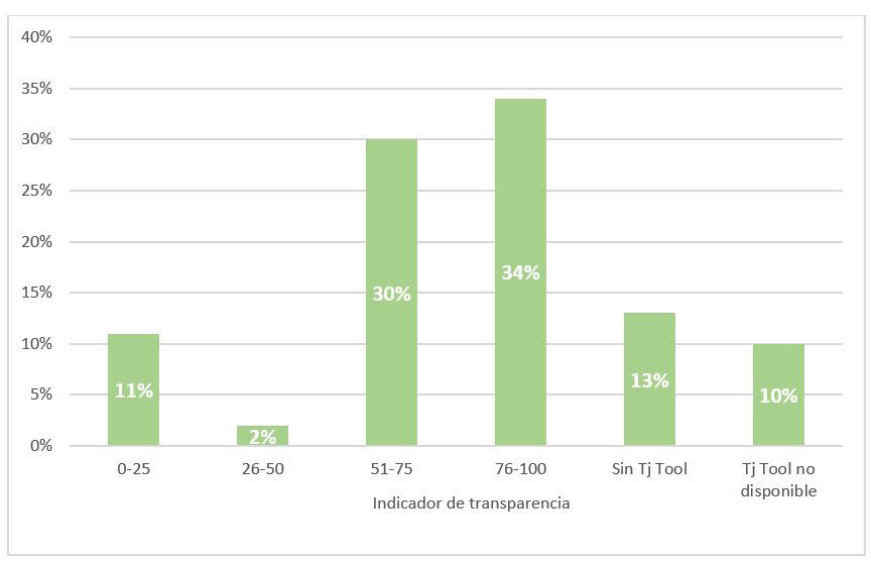

Gráfico 1. Porcentaje del indicador de transparencia en las noticias analizadas. Elaboración propia.

\subsection{Análisis de parámetros en relación con el indicador de transparencia}

En relación con las preguntas de investigación 2 y 3, una vez determinados los porcentajes del indicador de transparencia resultantes en las noticias objeto de estudio, estos se ponen en relación con los parámetros propuestos para su análisis calculados con base en los porcentajes de noticias incluidas en cada intervalo. En la tabla 2 se pueden observar los resultados obtenidos tras haber calculado los porcentajes aplicados a los parámetros Noticia propia/agencia y ponerlos en relación el porcentaje de noticias según su indicador de transparencia (En la tabla señalado como IT).

Se comprueba que en las noticias que obtienen entre el 76 y el $100 \%$ del valor del indicador de transparencia, que es el contiene el mayor porcentaje de las informaciones analizadas, prácticamente la totalidad de las noticias, un 98\%, son noticias propias. En las noticias que resultan entre un 51 y un 75\% del indicador de transparencia, también el mayor porcentaje de las informaciones son propias, aunque en este caso, el porcentaje es menor que en el anterior, de un $54 \%$. Solo en las noticias que no tienen la herramienta de transparencia, se encuentran menos informaciones de elaboración propia que noticias de agencia. Asimismo, destaca que la totalidad de las informaciones que no disponen de la herramienta de transpa-

\section{Ábalos anticipa la ampliación del estado de alarma: "No creo que en 15 días estemos en la capacidad de ganar esta batalla"}

Por su parte, el ministro de Interior Fernando Grande-Marlaska ha afirmado que el cierre de las fronteras exteriores españolas es "una poisibilidad real" para frenar al coronavirus.

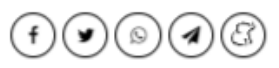

Figura 3. Cabecera de noticia sin el icono de la herramienta de transparencia. Fuente: Publico.es 


\section{Sánchez rinde cuentas sobre el estado de alarma en un Congreso vacío por el coronavirus}

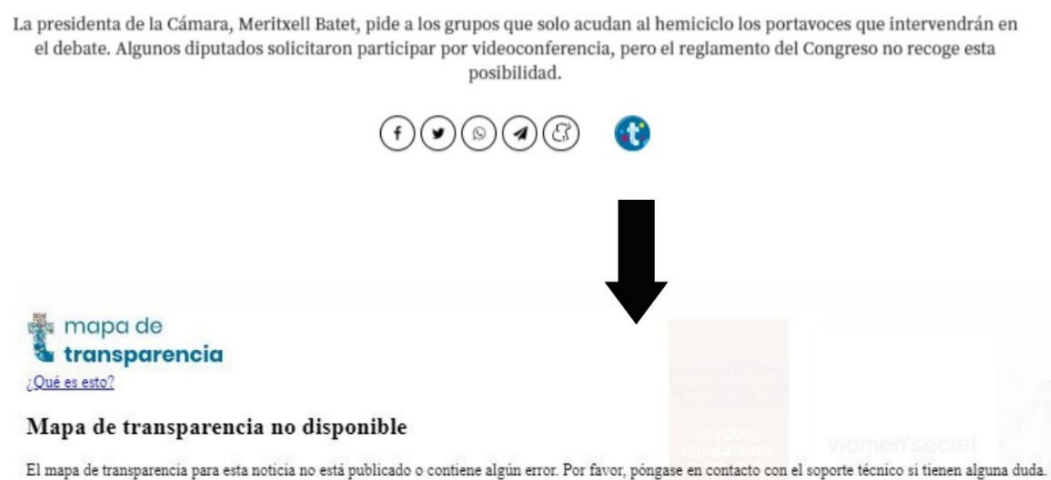

Figura 4. Cabecera de noticia con el icono de la herramienta de transparencia y con la herramienta no disponible. Fuente: Publico. es. Elaboración propia.

rencia son noticias propias.

Respecto a las noticias que cuentan con declaraciones del Gobierno o de la oposición los resultados se pueden observar en la tabla 3.

Así, se observa una mayor presencia de noticias con declaraciones de miembros del Gobierno en todos los intervalos, mientras que las noticias con declaraciones de la oposición tienen una presencia menor. De hecho, no superan el 20\% en ninguno de los intervalos. Destaca que las noticias que no incluyen declaraciones de miembros del Gobierno y de la oposición son mayoritarias con un $63 \%$ en las informaciones que no tienen la herramienta de transparencia, y presentan también un elevado porcentaje, un $40 \%$, en las noticias en las que la herramienta no está disponible.

Por lo que respecta a las palabras que aparecen en un mayor porcentaje en las noticias analizadas según su indicador de transparencia, la tabla 4 refleja que la palabra coronavirus es la palabra más repetida en el contenido de las informaciones analizadas, con porcentajes similares en todos los intervalos

\begin{tabular}{|l|l|l|l|}
\hline \multicolumn{1}{|c|}{$\begin{array}{c}\text { Porcentaje de } \\
\text { noticias según IT }\end{array}$} & $\begin{array}{c}\text { Noticias } \\
\text { Propias }\end{array}$ & $\begin{array}{c}\text { Noticias } \\
\text { de } \\
\text { agencia }\end{array}$ & $\begin{array}{c}\text { Propias y } \\
\text { Agencia }\end{array}$ \\
\hline 0-25: $11 \%$ & $58 \%$ & $42 \%$ & 0 \\
\hline 26-50: $2 \%$ & $50 \%$ & $50 \%$ & 0 \\
\hline $51-75: 30 \%$ & $54 \%$ & $27 \%$ & $19 \%$ \\
\hline 76-100: 34\% & $98 \%$ & $2 \%$ & 0 \\
\hline Sin IT: 13\% & $8 \%$ & $48 \%$ & $44 \%$ \\
\hline IT no disponible: $10 \%$ & $100 \%$ & 0 & 0 \\
\hline
\end{tabular}

Tabla 2. Resultados relación IT con Noticias Propias/ Agencia. Elaboración propia. estudiados.

En concreto, en el 39\% de las noticias que obtienen hasta un $25 \%$ en el indicador de transparencia, la palabra que más se repite es coronavirus, seguida de estado de alarma, con el 32\% de las informaciones objeto de estudio. En el caso de las noticias con un indicador de transparencia entre el 26 y el $50 \%$ y el 51 y $75 \%$, aproximadamente en la mitad de las informaciones analizadas (un 48\%), la palabra coronavirus es la que se ha encontrado más veces, seguida, por estado de alarma y COVID, respectivamente. En el siguiente intervalo, que corresponde a las noticias que resultan con más del $75 \%$ de indicador de transparencia, en más de la mitad de estas (un 52\%) predomina la palabra coronavirus. Aquí, estado de alarma supera en presencia a COVID en 7 puntos porcentuales. Se encuentra una diferencia de 11 puntos porcentuales entre las noticias sin herramienta de transparencia o que no disponen de ella en cuanto a las informaciones con mayor presencia de la palabra coronavirus, pero, en ambos casos, se mantiene como la palabra más repetida, con un 46 y un $55 \%$ de las noticias, respectivamente. En la tabla también se comprueba la menor presencia de las palabras cuarentena y confinamiento en

\begin{tabular}{|l|l|l|l|}
\hline $\begin{array}{c}\text { Porcentaje de } \\
\text { noticias según } \\
\text { IT }\end{array}$ & $\begin{array}{c}\text { Declaraciones } \\
\text { Gobierno }\end{array}$ & $\begin{array}{c}\text { Declaraciones } \\
\text { oposición }\end{array}$ & Resto \\
\hline 0-25: 11\% & $66 \%$ & $16 \%$ & $12 \%$ \\
\hline $26-50: 2 \%$ & $100 \%$ & 0 & 0 \\
\hline $51-75: 30 \%$ & $50 \%$ & $6 \%$ & $44 \%$ \\
\hline $76-100: 34 \%$ & $47 \%$ & $19 \%$ & $34 \%$ \\
\hline Sin IT: 13\% & $31 \%$ & $6 \%$ & $63 \%$ \\
\hline $\begin{array}{l}\text { IT no disponible: } \\
\text { 10\% }\end{array}$ & $50 \%$ & $10 \%$ & $40 \%$ \\
\hline
\end{tabular}

Tabla 3. Resultados relación IT con Declaraciones Gobierno/ Oposición. Elaboración propia. 


\begin{tabular}{|l|l|l|l|l|l|}
\hline Porcentaje de noticias según IT & \multicolumn{1}{|c|}{ Coronavirus } & \multicolumn{1}{|c|}{ COVID } & \multicolumn{1}{|c|}{ Estado de alarma } & \multicolumn{1}{c|}{ Cuarentena } & \multicolumn{1}{c|}{ Confinamiento } \\
\hline 0-25: $11 \%$ & $39 \%$ & $17 \%$ & $32 \%$ & $3 \%$ & 0 \\
\hline $26-50: 2 \%$ & $48 \%$ & $16 \%$ & $36 \%$ & $2 \%$ & $0 \%$ \\
\hline $51-75: 30 \%$ & $48 \%$ & $25 \%$ & $23 \%$ & $4 \%$ & $2 \%$ \\
\hline $76-100: 34 \%$ & $52 \%$ & $17 \%$ & $24 \%$ & $9 \%$ & $3 \%$ \\
\hline Sin IT: $13 \%$ & $46 \%$ & $17 \%$ & $21 \%$ & $2 \%$ & $7 \%$ \\
\hline IT no disponible: $10 \%$ & $55 \%$ & $23 \%$ & $19 \%$ & $1 \%$ \\
\hline
\end{tabular}

Tabla 4. Resultados según los términos encontrados en las noticias. Elaboración propia.

todos los intervalos del indicador de transparencia y también en las noticias que no disponen del mismo. Sin embargo, la presencia de la palabra "Confinamiento" destaca en el $9 \%$ de las noticias que tienen un indicador de transparencia menor de $25 \%$, siendo en este intervalo donde este porcentaje es mayor. La palabra "Cuarentena" tiene un comportamiento similar en las noticias que no tienen la herramienta de transparencia; en el $9 \%$ de las noticias analizadas predomina este término, superando al resto de intervalos estudiados.

\section{Discusión y conclusiones}

A continuación, se sintetizan los resultados de esta investigación y se responde a las preguntas de investigación que los han guiado. Con respecto a la primera pregunta de investigación (RQ1), referente al análisis del indicador de transparencia y los intervalos que aglutinan un mayor y menor porcentaje de noticias relacionadas con la COVID-19, los resultados señalan que la mayor parte de las informaciones obtienen un indicador de transparencia superior al 51\%. Sin embargo, ninguna noticia resulta con el $100 \%$ del indicador de transparencia y solo el 2\% alcanza el valor máximo obtenido, que es del 94\%. Por otro lado, al no encontrarse ninguna noticia con el $0 \%$ de transparencia, se puede afirmar que las informaciones del diario muestran, a través de la herramienta que permite la trazabilidad de las informaciones, que todas las noticias publicadas alcanzan un mínimo de transparencia que, en este caso, es de un $12 \%$. La cuestión se plantea al observar que en el $23 \%$ de las noticias no aparece o no se encuentra disponible la herramienta de transparencia. Este es un porcentaje superior al de las noticias cuyo indicador de transparencia no sobrepasa el $50 \%$, que es, concretamente, de un 13\%. En este sentido, se puede afirmar que la herramienta aporta ese valor de transparencia y veracidad en sus informaciones, pero no en todas ellas.

Esta afirmación enlaza con las preguntas de investigación 2 y 3 (RQ1 y RQ2), que se refieren a la relación entre los indicadores de transparencia de las noticias y los parámetros analizados, así como la correlación entre dichos parámetros y las informaciones en las que no es posible acceder al indicador de transparencia. Existen diferencias significativas entre los distintos parámetros que diversifican su relación con las noticias en los intervalos de indicadores de transparencia establecidos. En primer lugar, las noticias relacionadas con la COVID-19 en el período objeto de estudio que cuentan con un mayor indicador de transparencia (superior al 76\%) son las noticias de elaboración propia, mientras que las noticias con menos del 76\% de indicador de transparencia muestran porcentajes más equilibrados entre noticias propias y de agencia. Por tanto, se establece una primera relación entre las noticias propias y un mayor indicador de transparencia. En las noticias que no disponen de la herramienta, solo un $8 \%$ de las noticias propias no la presenta, pero todas las informaciones que no tienen la herramienta disponible son noticias propias. De esta manera, se observa que las noticias propias implican un mayor indicador de transparencia, pero también que la herramienta de transparencia no esté disponible.

En segundo lugar, en las noticias relacionadas con la COVID-19 en el período estudiado se encuentra una mayor presencia de las noticias con declaraciones del gobierno en todos los intervalos. Esto sucede en gran medida debido a que son más las noticias en las que se encuentran declaraciones de miembros del Gobierno que de la oposición. Sí se puede establecer una relación con las noticias en las que no aparece la herramienta de transparencia que corresponden en un 63\% a noticias en las que no hay declaraciones de Gobierno ni oposición.

Por otra parte, los resultados de los términos analizados arrojan varias conclusiones. En una primera aproximación a los datos, se percibe que la palabra "coronavirus" es la que más se repite en todos los intervalos del indicador de transparencia, con un porcentaje similar. Si bien se puede destacar que es en las noticias con un indicador de transparencia superior al 76\% en las más aparece. En este sentido, se puede establecer una relación entre un mayor indicador de transparencia y una mayor presencia de la palabra "coronavirus". De hecho, son menos las noticias con la palabra coronavirus (un 39\%) las noticias por debajo del $25 \%$ resultante de este indicador. Respecto a la palabra COVID, se encuentra relacionada con las noticias con un mayor indicador de transparencia, presentando una menor incidencia en las noticias con un indicador de Transparencia por debajo del 50\%. En el caso de "cuarentena", dado que los valores son bajos en todos los intervalos, la relación se vislumbra en las noticias que no tienen la herramienta de transparencia, ya que en el $9 \%$ de estas informaciones se repite la palabra "cuarentena" por encima de los demás términos estudiados. Al poner atención en "confinamiento", la 
incidencia en las noticias de esta palabra es baja, sin embargo, destaca en las informaciones con un índice de transparencia menor del 25\%. De esta manera, se puede establecer una relación entre las noticias con un menor resultado en la herramienta de transparencia y una mayor incidencia de esta palabra en las informaciones.

Con todo lo analizado anteriormente, cabe interpretar los datos con la perspectiva de una situación inédita como es la declaración de estado de alarma en un contexto de pandemia mundial provocada por el virus de la COVID-19. Como se ha mencionado, es un momento en el que los medios digitales experimentan un aumento en su consumo, pero también se produce una mayor circulación de "bulos" y noticias falsas, por lo que es importante disponer de elementos que garanticen la veracidad de las informaciones. Además de las plataformas de fact checking que permiten contrastar informaciones publicadas en medios digitales y que circulan en redes sociales, el diario Público.es dispone de la herramienta de transparencia para que el usuario pueda comprobar la trazabilidad de las informaciones que publica. En este sentido, en el presente estudio se ha comprobado que la mayor parte de las noticias obtienen un indicador de transparencia por encima del 50\%, siendo el 76\% el resultado más obtenido. Sin embargo, en un $23 \%$ de las noticias no se puede acceder a este indicador. La ausencia del indicador de transparencia se produce fundamentalmente en noticias de agencia, mientras que la no disponibilidad de éste se da en noticias propias. Otro dato reseñable es la presencia frecuente de Declaraciones de gobierno, aunque no se puede establecer una correlación con las informaciones que obtienen un determinado indicador de transparencia. Sí se constata que la palabra "coronavirus" es la más usada en las noticias, sobre todo en las que tienen un indicador de transparencia más elevado.

A modo de conclusión, se menciona que en la presente investigación no se pierde de vista que la herramienta de transparencia responde a unos indicadores implementados por un programa informático con la finalidad de hacer públicos los procesos informativos y su trazabilidad. En este sentido, cabe destacar que, en el momento de realizar esta investigación, el diario Publico es el único medio digital en España que cuenta con una herramienta que muestra la trazabilidad de las informaciones, lo cual se considera como una muestra de la voluntad del diario por dotar de transparencia y credibilidad a las informaciones que publica. Se entiende que este hecho resulta de gran importancia en un contexto de viralización de contenidos falsos y de desconfianza de la sociedad hacia los medios de comunicación tradicionales como fuentes principales de información (Pérez-Curiel y Velasco-Molpeceres, 2020). Por lo que respecta a la herramienta informática, se considera que debería estar operativa en la totalidad de las noticias que aparecen publicadas en el periódico, para ofrecer una completa seguridad y confianza al usuario que accede a las informaciones vertidas por el diario.

El objetivo de este estudio es analizar este indicador de acuerdo con unos parámetros concretos en un espacio de tiempo determinado por la pandemia de la COVID-19. En este sentido, se considera que los límites de la investigación vienen determinados por la posibilidad de realizar un estudio comparativo con los mismos parámetros en otro período del estado de alarma o poniendo el foco en los ocho indicadores del mapa de transparencia que dan como resultado el porcentaje final que corresponde a cada noticia. Sin embargo, estas cuestiones se plantean como objeto de futuras investigaciones relacionadas con esta herramienta de trasparencia implementada por el diario Publico.es.

Asimismo, tras el estudio llevado a cabo, es posible afirmar que en un contexto incierto como es una pandemia mundial en el que el acceso a la información veraz es de vital importancia para la sociedad, además de la presencia de verificadores de la información y de las herramientas de transparencia que puedan ir implementando los diferentes medios de comunicación, es necesaria la formación en nuevas competencias que se adapten al impacto de la innovación tecnológica, tal y como proponen Besalú et ál. (2017) y Casero-Ripollés et ál. (2013). En un momento de sobrecarga informativa por diferentes canales como son los medios convencionales y también las redes sociales, se ha de dotar a la ciudadanía de herramientas para poder discernir entre aquello que es veraz de aquello que no lo es, así como apelar a la propia responsabilidad de la sociedad en cuanto a no difundir contenidos de dudosa veracidad, avisar de la circulación de noticias falsas y contribuir a reducir la desinformación (Palomo y Sedano, 2018). Por ello, se ha de avanzar en la implantación de las herramientas de verificación y transparencia de datos y en la alfabetización ciudadana en el actual escenario de sobresaturación informativa en un entorno de pandemia mundial.

\section{Referencias}

Abuín-Vences, N., Sierra-Sánchez, J., Mañas-Viniegra, L., y NúñezGómez, P. (2020). Tratamiento informativo de la pandemia del coronavirus en los medios digitales españoles. Hipertext.net, (21), 15-26. https://doi.org/10.31009/hipertext.net.2020.i21.02

Aleixandre-Benavent, R., Castelló-Cogollos, L., y Valderrama-Zurián, J.-C. (2020). Información y comunicación durante los primeros meses de Covid-19. Infodemia, desinformación y papel de los profesionales de la información. El Profesional de la Información, 29(4). https://doi. org/10.3145/epi.2020.jul.08

Amorós-Garcia, M. (2018). Fake News. La verdad de las noticias falsas. Plataforma Editorial.

Aparici, R., y García-Marín, D. (2019). La posverdad. Una cartografía de los medios, las redes y la política. Gedisa.

Appelgren, E., y Salaverría, R. (2018). The promise of the transparency culture: A comparative study of access to public data in Spanish and Swedish newsrooms. Journalism practice, 12(8), pp. 986-996. https:// doi.org/10.1080/17512786.2018.1511823 
Ayuso, B., y Bauzá, B. (2018). Almanaques, espías y un chándal: el universo secreto de los fact-checkers. Jot Down, (31), 4-13.

Bergström, A., y Jervelycke, M. (2018). News in Social Media. Digital Journalism, 6(5), pp. 583-598. https://www.doi.org/10.1080/2167081 1.2018 .1423625

Besalú, R., Schena, J., y Sánchez, C. (2017). Competencias más relevantes en los estudios de Comunicación Audiovisual. Las percepciones de profesionales, docentes y estudiantes. Revista Latina de Comunicación Social, (72), pp. 1.536-1.553. http://dx.doi. org/10.4185/RLCS-2017-1233

Campos-Domínguez, E., y Redondo-García, M. (2015). Meta periodismo y transparencia informativa en el periodismo del siglo XXI. Obets. Revista de ciencias sociales, 10(1), pp. 185-209. https://doi. org/10.14198/OBETS2015.10.1.07

Casero-Ripollés, A. (2020). Impacto del Covid-19 en el sistema de medios. Consecuencias comunicativas y democráticas del consumo de noticias durante el brote. El Profesional de la Información, 29(2). https://doi.org/10.3145/epi.2020.mar.23

Casero-Ripollés, A., Ortells Badenes, S., y Doménech Fabregat, H. (2013). Las competencias profesionales en periodismo: una evaluación comparativa. Historia y Comunicación Social, (18), pp. 53-64. https://doi.org/10.5209/rev_HICS.2013.v18.44311

Cherubini, F., y Graves, L. (2016). The rise of fact-checking sites in Europe. Reuters Institute for the Study of Journalism, University of Oxford.

Culver, S., y Jacobson, T. (2012). Alfabetización mediática como método para fomentar la participación cívica. Comunicar, 20(39), pp. 73-80. https://doi.org/10.3916/C39-2012-02-07

Diakopoulos, N., y Koliska, M. (2017). Algorithmic transparency in the news media. Digital journalism, 5(7), pp. 809-828. https://doi.org/10.1 080/21670811.2016.1208053

Derakhshan, H., y Wardle, C. (2017). Information Disorder: Definitions. En Understanding and Addressing the Disinformation Ecosystem (pp. 5-12). Annenberg School for Communication. http://bit.ly/32F7v5n

Díez-Garrido, M., Campos-Domínguez, E. (2018). Los periodistas españoles ante la transparencia en España: Valoración y uso de la apertura informativa. Revista española de la transparencia, 7, 49-69. https://uvadoc.uva.es/handle/10324/42107

Dizikes, P. (2018). Study: On Twitter, false news travels faster than true stories. MIT News Office. https://bit.ly/2toeBNS

Duke Reporter's Lab. (2019). Fact-Checking. http://bit.ly/2YrCBft

European Commision (2018a). Flash Eurobarometer 464. Fake news and disinformation online. http://bit.ly/2wSH3t8

European Commision (2018b). A multi-dimensional approach to disinformation. Report of the independent High level Group on fake news and online disinformation. https://bit.ly/37TYo2T

Fabry, M. (24 de agosto de 2017). Here's How the First Fact-Checkers Were Able to Do Their Jobs Before the Internet. Time. https://bit. ly/35FEdnA

Fengler, S., Eberwein, T., Leppik-Bork, T., Lönnendonker, J., Pies, J. (2014). Introduction: media accountability - Basic concepts and theoretical foundations. En S. Fengler, Eberwein, T., Mazzoleni, G., Porlezza, C. (eds). Journalists and media accountability. An international study of news people in the digital age, (pp 7-30). Peter lang. https://doi.org/10.3726/978-1-4539-1247-8
García-Avilés, J. A. (2017). [Editorial] Periodismo digital en la Sociedad Red: innovar en un entorno competitivo. Revista Mediterránea de Comunicación, 8(1), pp. 3-5. https://doi.org/10.14198/MEDCOM.10336

García-Galera, M. C., Del-Hoyo-Hurtado, M., y Blanco-Alfonso, I. (2020). Desinformación e intención comunicativa: una propuesta de clasificación de fake news producidas en entornos periodísticos profesionales. Revista Mediterránea de Comunicación/Mediterranean Journal of Communication, 11(2), pp. 105-118. https://www.doi. org/10.14198/MEDCOM2020.11.2.16

Gueham, F. (2017). Le fact-checking: une réponse à la crise de l'information et de la démocratie. Fondation pour l'innovation politique.

Heikkilä, H., Domingo, D., Pies, J., Gtowacki, M., Kuś, M., Baisnée, O. (2012). Media accountability goes online: a transnational study on emerging practices and innovations. Journalism Research and Development Centre. https://bit.ly/3121AIS

IAB Spain. (2020). Estudio de Redes Sociales 2020. IAB Spain.

Ireton, Ch., y Posetti, J. (2018). Journalism, 'fake news' \& disinformation: handbook for journalism education and training. Unesco Publishing. https://bit.ly/340oKSY

Keyes, R. (2004). The Post-truth Era: Dishonesty and deception in contemporary life. St. Martins Pr.

Magallón Rosa, R. (2018). La biblioteca digital sobre Donald Trump. Fact-checking frente a fake news. Estudios sobre el Mensaje Periodístico 24(1), 273-282. https://doi.org/10.5209/ESMP.59949

Mantzarlis, A. (2018). Fact-checking 101. En Ireton, C., y Posetti, J. (Eds.), Journalism, fake news $\&$ disinformation: Handbook for journalism education and training, (pp. 81-95). Unesco.

Mapa de transparencia, (2020). TJ Tool la herramienta para crear el mapa de transparencia de la información. Público.es. http://bit. ly/2Yv4vr7

Molina-Cañabate, J., y Magallón-Rosa, R. (2020). Desinformación y periodismo científico. El caso de Maldita Ciencia.. Revista Mediterránea de Comunicación, 11(2), pp. 11-21. https://doi.org/10.14198/ MEDCOM2020.11.2.4

Nielsen, R. K., y Graves, L. (2017). News you don't believe: Audience perspectives on fake news. Reuters Institute for the Study of Journalism. Oxford University. https://bit.ly/2XdRCBD

Negredo, S., Amoedo, A., Vara-Miguel, A., Moreno, E., y Kaufmann, J. (2020). Digital news report.es 2020. Gobiernos, políticos y redes sociales, principales responsables del auge de los bulos. Center for Internet Studies and Digital Life. Universidad de Navarra.

Nigro, P. (2018). Causas De La pérdida de la confianza en la prensa y estrategias para su restablecimiento en un contexto de incertidumbre. Hipertext.net, (17), 54-63, https://doi.org/10.31009/hipertext. net.2018.i17.05

Nyhan, B., y Reifler, J. (2012). Misinformation and fact-checking: Research findings from social science. New America Foundation Media Policy Initiative Research Paper. http://bit.ly/35rTeJf

OMS (2 febrero 2020). Rolling updates on coronavirus disease (Covid19). https://bit.ly/3pzhfsx

Orbegozo-Terradillos, J., Morales-i-Gras, J., \& Larrondo-Ureta, A. (2020). Desinformación en redes sociales: ¿compartimentos estancos o espacios dialécticos? El caso Luther King, Quim Torra y El Confidencial. Revista Mediterránea de Comunicación, 11(2), 55-69. https://doi.org/10.14198/MEDCOM2020.11.2.2 
Palau-Sampio, D. (2018). Fact-checking y vigilancia del poder: La verificación del discurso público en los nuevos medios de América Latina. Communication \& Society, 31(3), 347-363. https://doi. org/10.15581/003.31.3.347-363

Palomo, B., y Sedano Amundarain, J. A. (2018). WhatsApp como herramienta de verificación de fake news. El caso de B de Bulo. Revista Latina, (73), 1384-1397. https://doi.org/10.4185/RLCS-2018-1312

Pérez-Curiel, C., y Velasco Molpeceres, A. M. (2020). Impacto del discurso político en la difusión de bulos sobre Covid-19. Influencia de la desinformación en públicos y medios. Revista Latina, (78), 86-119. https://doi.org/10.4185/RLCS-2020-1469

Pérez Escoda, A. (2018). Uso de smartphones y redes sociales en alumnos/as de Educación Primaria. Revista Prisma Social, (20), 76-91. https://revistaprismasocial.es/article/view/2310

Ramon-Vegas, X., Mauri-Ríos, M., y Rodríguez-Martínez, R. (2020). Redes sociales y plataformas de fact-checking contra la desinformación sobre la COVID-19. Hipertext.net, (21), 79-92. https://doi. org/10.31009/hipertext.net.2020.i21.07

Sánchez, S. (2019). Tj Tool: una herramienta que busca aumentar la transparencia del periodismo midiendo su trazabilidad. IJNet Red Internacional de Periodistas. https://bit.ly/3alezRY

Salaverría, R., Buslón, N., López-Pan, F., León, B., López-Goñi, I., y Erviti, M.-C. (2020). Desinformación en tiempos de pandemia: tipología de los bulos sobre la Covid-19. El Profesional de la Información, 29(3). https://doi.org/10.3145/epi.2020.may.15

Silverman, C., Strapagiel, L., Shaban, H., y Hall, E. (2016). Hyperpartisan Facebook pages are publishing false and misleading information at an alarming rate. Buzzfeed News. https://bzfd.it/2eKWOEi

Stewart, A., Mosleh, M., Diakonova, M., Arechar, A., Rand, D., y Plotkin, J. (2019). Information gerrymandering and undemocratic decisions. Nature: International Weekly Journal of Science, 573(7772), 117-121. http://dx.doi.org/10.1038/s41586-019-1507-6

Suárez-Villegas, J. C., Rodríguez-Martínez, R., Ramon-Vegas, X. (2020). Pluralismo informativo en la era de la deliberación digital: percepciones de periodistas y ciudadanos. Profesional de la información, 29(5). https://doi.org/10.3145/epi.2020.sep.25

Terol-Bolinches, R., y Alonso-López, N. (2020). La prensa española en la Era de la Posverdad: el compromiso de la verificación de datos para combatir las Fake News. Revista Prisma Social, (31), 304-327. http://bit.ly/2MqjlfG

Ufarte-Ruiz, M. J., Anzera, G., y Murcia-Verdú, F. J. (2020). Plataformas independientes de fact-checking en España e Italia. Características, organización y método. Revista Mediterránea de Comunicación, 11(2), pp. 23-39. https://www.doi.org/10.14198/MEDCOM2020.11.2.3
Ufarte-Ruiz, M. J., Sidorenko-Bautista, P., y Cantero de Julián, J. I. (2019). El negocio de las noticias falsas. El caso de El Mundo Today. Historia y comunicación social, 24(2), 561-578. https://doi. org/10.5209/hics.66300

Zommer, L. (2014). El boom del fact checking en América Latina. Aprendizajes y desafíos del caso de Chequeado. Konrad Adenauer Stiftung.

\section{CV}

Nadia Alonso-López. Doctora en Comunicación, Máster en Gestión de la Producción Audiovisual, Máster en Marketing y Comunicación Digital. Desarrolla su labor docente e investigadora en la Universitat Politècnica de València, donde es profesora en el Grado de Comunicación Audiovisual. Forma parte del grupo de investigación ArtiCom y es miembro de la Asociación Española de Investigación de la Comunicación (AE-IC). Es autora de diversos artículos de investigación, capítulos de libro y aportaciones en congresos sobre consumo audiovisual, redes sociales, desinformación y mecanismos de verificación, transmedia y educación. Tiene una experiencia profesional de más de 20 años en medios de comunicación como periodista, presentadora y guionista en informativos, documentales y proyectos de ficción.

Raúl Terol Bolinches. Doctor en Industrias Culturales y de la Comunicación por la Universitat Politècnica de Valencia. En la actualidad es profesor del Grado de Comunicación Audiovisual, en el Campus de Gandia de esta misma universidad, impartiendo las asignaturas de Proceso de Realización Radiofónica e Historia de los Medios Audiovisuales. Por lo que respecta a investigación, Terol ha realizado diversas aportaciones en congresos, así como en publicaciones impresas y capítulos de libro, sobre el estado actual de la comunicación radiofónica y la presencia del sonido en Internet. También ha trabajado e impartido cursos de formación para profesorado en el Instituto de Ciencias de la Educación de la UPV, sobre uso del pódcast y las redes sociales al servicio de la docencia. Al margen del aspecto académico, Raúl Terol presenta diariamente un espacio radiofónico en la emisora regional 99.9 Valencia Radio y fue director de la radio municipal Llosa FM.

\section{осм Observatorio de Cibermedios}

\section{https://observatoriocibermedios.upf.edu/}

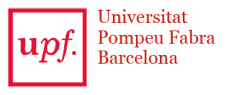

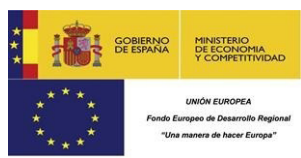

El Observatorio de Cibermedios es una producción del Grupo de Investigación en Documentación Digital y Comunicación Interactiva (DigiDoc) del Departamento de Comunicación de la Universitat Pompeu Fabra.

El Observatorio de Cibermedios (OCM) forma parte del proyecto del Plan Nacional "Narración interactiva y visibilidad digital en el documental interactivo y el periodismo estructurado". RTI2018-095714-B-C21 (MINECO/FEDER), Ministerio de Ciencia, Innovación y Universidades (España). 